\title{
Cockroach as a Vector of Blastocystis sp. is Risk for Golden Monkeys in Zoo
}

\author{
Lei Ma1,* ${ }^{10}$, Yongbin Zhang'2, Haixia Qiao', Shuai Li', Heqin Wang', Ningning Zhang ${ }^{1}$, Xiao Zhang ${ }^{3}$ \\ ${ }^{1}$ College of Life Science, Hebei Normal University, Shijiazhuang 050024, Hebei Province, China; ${ }^{2}$ Shijiazhuang Municipal Administration Center of \\ Zoo, Shijiazhuang 050200, Hebei Province, China; ${ }^{3}$ Department of Preventive Veterinary Medicine, College of Veterinary Medicine, \\ Shandong Agricultural University, Taian City 271000, Shandong Province, China
}

\begin{abstract}
Blastocystis sp. is a kind of protozoa living in the intestinal tract of human and animals, which will cause intestinal diseases such as diarrhea, abdominal distension and vomiting. This paper was aimed to understand the infection of Blastocystis sp. In golden monkeys and the transmission path in North China. Thirty-seven feces samples from golden monkeys and 116 cockroach samples from Shijiazhuang Zoo were collected from July to October 2019 for PCR analysis of Blastocystis sp. Genetic diversity analysis was further conducted on the samples with positive PCR results. The results showed that the infection rate was $48.7 \%$ (18/37) in golden monkeys and $82.8 \%(96 / 116)$ in cockroaches, respectively. The genetic evolution analysis based on small subunit ribosomal RNA demonstrated that three subtypes (ST) of Blastocystis sp. including ST1, ST2, and ST3 existed in the intestinal tract of golden monkeys, while only ST2 was detected in the intestinal tract of cockroaches. This paper may provide supports for the quarantine and control of Blastocystis sp. for the zoo in Northern China.
\end{abstract}

Key words: Blastocystis sp., zoonosis, golden monkey, cockroach, gene subtype

Blastocystis sp. is a zoonotic parasite belonging to the phylum Protozoa. It lives in the intestinal tracts of humans and primates and is widely distributed around the world. It will result in gastrointestinal symptoms such as diarrhea, abdominal pain and vomiting, which will affect the health and growth of the host $[1,2]$. Recently, it has been demonstrated that people with low immunity, HIV/AIDS patients and patients with intestinal stress syndrome after infected with Blastocystis sp. present obvious symptoms of diarrhea and abdominal pains among children [3,4]. In addition, Blastocystis sp. infection is also closely related to repeated abdominal pain in children [5]. Above findings have attracted attentions from many scholars on the pathogenic potential of Blastocystis sp. Currently, the reported Blastocystis sp. hosts include livestock (cattle, sheep, camel, pig, etc.), poultry (chicken, duck, etc.) and wild animals (panda, fox, boar, sika deer, goral, peacocks, swan, etc.). Among those, nonhuman primates have been widely paid attention on, because they are susceptible to the Blastocystis sp.

\footnotetext{
- Received 25 April 2020, revised 23 September 2020, accepted 24 September 2020. *Corresponding author (Imahappy@hebtu.edu.cn) (C) 2020, Korean Society for Parasitology and Tropical Medicine This is an Open Access article distributed under the terms of the Creative Commons Attribution Non-Commercial License (https://creativecommons.org/licenses/by-nc/4.0) which permits unrestricted non-commercial use, distribution, and reproduction in any medium, provided the original work is properly cited.
}

and have the closest genetic relationship with humans. It has been reported worldwide that nonhuman primates such as chimpanzees, mandrills, macaques and baboons, can be infected by Blastocystis sp., and understanding Blastocystis sp. is very important for the public health.

The small subunit ribosomal RNA (SSU rRNA) genetic analysis showed that there are at least 26 subtypes (STs) of Blastocystis sp. [6]. However, different STs exhibit significant distribution differences in different animal hosts. For example, 10 STs including ST1-9 and ST12 can infect humans, among which ST1-4 are more common; at least 11 STs such as ST1-5, ST7, ST8, ST10, ST13, ST15, and ST19 can infect nonhuman primates; other STs are most observed in nonhuman primates [710]. In addition, the distribution of Blastocystis sp. also demonstrates geographical differences. In the case of zoonotic STs, ST3 is the most common in Europe, followed by ST4 and ST1, while ST3 is the most common in other regions, followed by ST1 and ST2 [11-13].

So far, no report has been disclosed about human and animal infection by Blastocystis sp. in Northern China [14]. Golden monkey as one of the unique species in China, has been introduced by the zoos in various provinces because of its great ornamental value and popularity. Thus, golden monkeys were considered as the subjects in this study. Given the lack of 
data about the life history, transmission path and transmission mechanism of Blastocystis sp., this study adopted molecular biology method based on SSU rRNA gene to investigate the Blastocystis sp. infection rates of both the golden monkeys and cockroaches living in the same environment. The genetic diversity was analyzed to understand the transmission path of Blastocystis sp., which will lay the foundation for epidemiological analysis of Blastocystis sp.

Thirty-seven feces samples of golden monkeys were collected from Shijiazhuang Zoo in Hebei Province, and 116 cockroaches were also collected from the living area of the golden monkeys from July to October 2019. The feces samples were immediately transferred to the disposable sterile centrifuge tube after defecation and stored in refrigerator. After dissection, the cockroach intestines were taken out and stored in the refrigerator. This study complied with the guidelines of the Regulations for the Administration of Affairs Concerning Humans and Experimental Animals and was approved by the Biomedical Ethical Committee of Hebei Normal University, Hebei, China (ID: 2019SC14).

DNA from feces was purified using the Stool Genomic DNA Extraction Kit (Beijing Solarbio Science \& Technology Co., Ltd, Solarbio, Beijing, China). Polymerase chain reaction (PCR) amplification was performed according to the identification method of Blastocystis sp. based on SSU rRNA gene reported by Scicluna et al. [15]. The forward primer BhRDr (5'-GAGCTTTTTAACTGCAACAACG-3') and the reverse primer RD5 (5'-ATCTGGTTGATCCTGCCAGT-3') were used in a standard PCR reaction. Electrophoresis of PCR products was conducted in Goldviewcontaining agar gel, and the results were observed directly by UV gel imaging system. The amplified products with expected DNA fragment size were purified according to the specification of DNA Purification Kit (Solarbio ${ }^{\circledR}$ ) and sent to Shanghai Bioengineering Co., Ltd for sequencing.

PCR results showed that 18 golden monkey feces samples were successfully amplified with the expected size of the gene fragments, which were identified as Blastocystis sp. by sequencing. Thus, the infection rate for golden monkeys was $48.7 \%$ (18/37). In recent years, the infection rate of nonhuman primates by Blastocystis sp. in China has been always high. In 2010, Lv et al. [16] reported the infection rate of Rhinopithecus roxellana was 66.7\% in Shanxi Province, China. In 2017, Zhao et al. [10] claimed that the infection rates of Rhinopithecus roxellana, Cercopithecus neglectus, Macaca mulatta and Presbytis francoisi were $89.1 \%, 80.0 \%, 96.6 \%$, and $100.0 \%$, respectively, in
Qinling areas in China. In fact, He et al. found that a variety of non-human primates were infected by Blastocystis sp. in Guangzhou, China, and the highest rate could reach $78.9 \%$ as early as 1990 . Although the infection rate of $40.9 \%$ for golden monkeys investigated in this paper is much lower than the above reports, the probability that other primates are infected with this parasite cannot be ignored, let alone the impact of this parasite on the animal health. In addition, although there have been no reports about infection of golden monkeys by Blastocystis sp., the infection of non-human primates by the parasite have been widely reported. For example, the infection rate of Thai long-tailed macaques was $41.9 \%$ [17]; that of Ecuadorian howler monkeys was $60.0 \%$ [18]; those of Tanzanian chimpanzees, grivet monkeys and guereza monkeys were $71.4 \%, 84.7 \%$, and $83.7 \%$, respectively [19]; that of Nepalese macaques was $100.0 \%$ [20]; and the overall infection rate of Australian nonhuman primates was $37.9 \%$ [21]. Above results indicated that nonhuman primates are susceptible animals easily infected by Blastocystis sp., which is very common.

Cockroach, as a common indoor arthropod, can transmit a variety of pathogens, such as Blastocystis sp. However, just a few reports have illustrated the transmission of Blastocystis sp. by cockroaches. In the study, 96 cockroach samples were identified as Blastocystis sp., revealing that the infection rate was $82.8 \%$ (96/116) (Table 1). In 1993, Zaman et al. [22] first found Blastocystis sp. in cockroaches collected from a sewage pool in Singapore, which exhibited a high infection rate; and the existence of cysts in their isolates indicated that cockroaches may be the transmission media of Blastocystis sp. However, the infection rates in the following studies are significantly different from each other. Suresh et al. [23] reported a low infection rate $(10.0 \%)$ of cockroach in residential areas in Malaysia, while Farah Haziqah et al. [24] revealed an infection rate of $40.4 \%$ in cockroaches living in drainage systems in Malaysia after nearly a decade. The results in this study showed that the infection rate of cockroaches in the living area for golden monkeys was as high as $82.8 \%$, which is in sharp contrast to previous reports. The difference may be related to the host ani-

Table 1. Prevalence of Blastocystis sp. in golden monkeys and cockroaches

\begin{tabular}{lccccc}
\hline \multirow{2}{*}{ Hosts } & \multirow{2}{*}{$\begin{array}{c}\text { No. of } \\
\text { examined }\end{array}$} & No. of & \multicolumn{3}{c}{ Blastocystis sp. subtype (\%) } \\
\cline { 5 - 6 } & positive & ST1 & ST2 & ST3 \\
\hline Golden monkeys & 37 & 18 & 38.9 & 44.4 & 16.7 \\
Cockroaches & 116 & 96 & 0 & 100 & 0 \\
\hline
\end{tabular}


mals grown in different geographical environments.

The DNA sequences of the SSU rRNA genes were subjected to correction prior to BLAST alignment (https://blast.ncbi. nlm.nih.gov/Blast.cgi). The reference sequences (MK416177.1, KU361313.1, LT222069.1, KP055707.1, MN338075.1, KM374613.1, MF072955.1, KY610164.1, MN736506.1, KX234596.1 MK861933.1 and Proteromonas lacertae) used were from National Center for Biotechnology Information (NCBI, https://www.ncbi.nlm.nih. gov/) and Blastocystis sp. subtypes identified in the present study are indicated using conventional subtype designations. All seauences were aligned using MEGA 6.06 and edited manually (available on request). Bayesian analysis was performed using MrBayes 3.0. The analysis of 18 positive golden monkey samples showed that there were three genotypes, i.e. ST1, ST2, and ST3, accounting for 38.9\% (7/18), 44.4\% (8/18), and $16.7 \%$ (3/18), respectively. There was no dominant ST as shown in Fig. 1 and Table 1. But all the genotypes in 96 positive cockroach samples were ST2 (Table 1; Fig. 1). ST2 exists in both golden monkeys and cockroaches and their relationship were near with each other. Although there are few reports about cockroaches as the transmission media of Blastocystis sp., golden monkeys and cockroaches share the same Blastocystis sp. ST2, which is the dominant ST as transmitted by cockroaches and the possibility of mutual transmission between the 2 animals
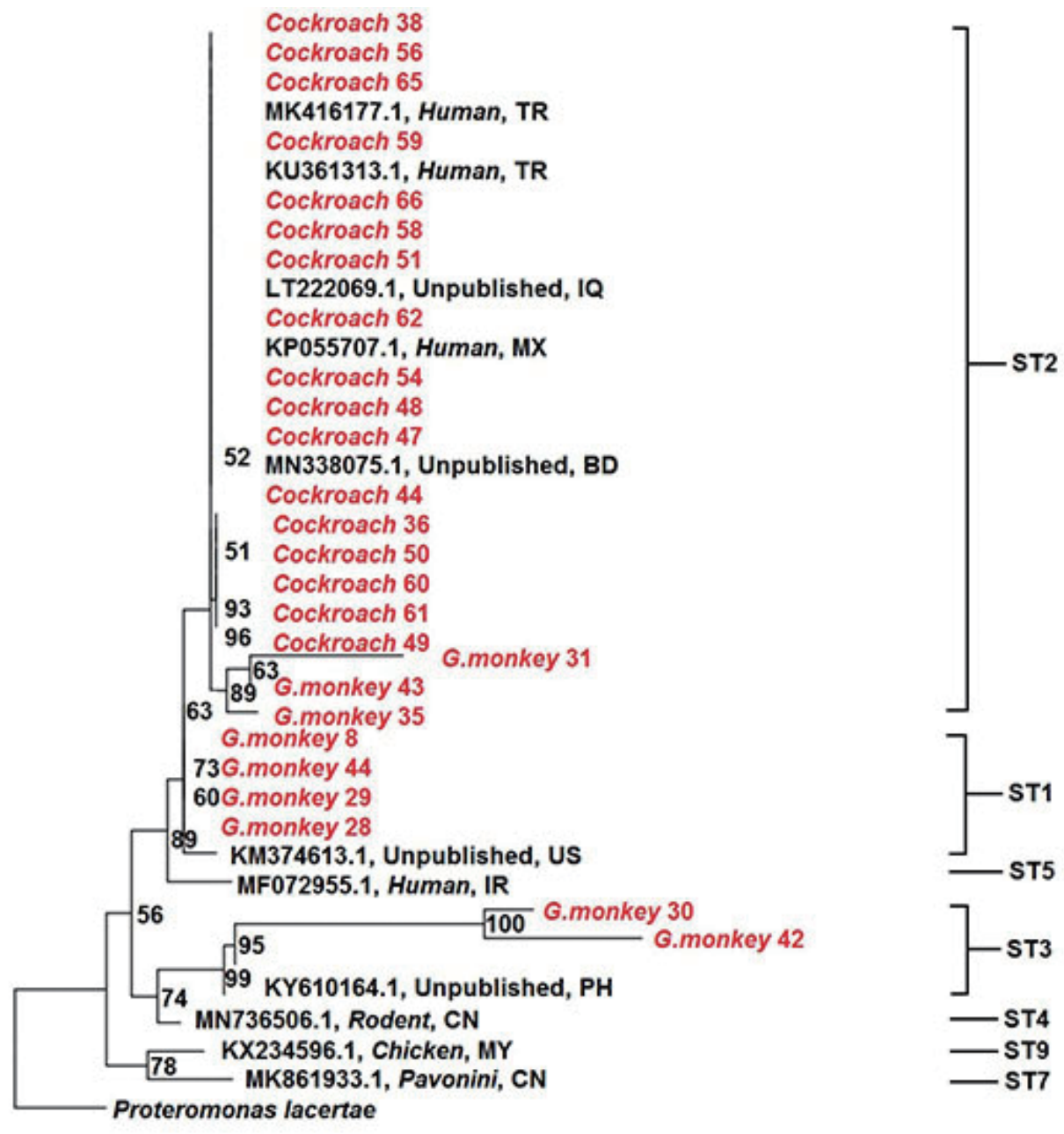

$\overline{0.07}$

Fig. 1. Phylogenetic relationships of subtypes of Blastocystis sp. based on the SSU rRNA in golden monkeys and cockroaches. Branch numbers represent percent bootstrapping values from 1,000 replicates. The Blastocystis sp. subtypes in this study were red. ST, subtype; G. monkey, Golden monkey; CN, China; TR, Turkey; IQ, Iraq; MX, Mexico; BD, Bangladesh; US, United States; IR, Iran; PH, Philippines; MY, Malaysia. 
cannot be excluded. The large-area activity of cockroaches may increase the transmission rate of Blastocystis sp. to other animals.

This study demonstrated that Blastocystis sp. as a zoonotic intestinal parasitic protozoa, exhibits different ST distribution in different hosts. Nonhuman primates with the closest genetic relationship with humans, are more likely to transmit the Blastocystis sp. to humans. Especially when the humans visited the zoo, the possibility will be increased too much for close contact between humans and the nonhuman primates, which will undoubtedly bring adverse effect on the human health. From the latest reports, the common zoonotic STs are ST1-ST9 and ST12 [25]. And ST2 is also considered as one of the more popular Blastocystis sp. STs in China, the role of cockroaches in the transmission cannot be ignored.

\section{ACKNOWLEDGMENTS}

This work was supported by China Postdoctoral Science Foundation (No. 2019M651061) and the Foundation of Hebei Normal University (No. L2018B10). All authors participated in the collection of samples, and performed the laboratory tests and analysis. Ma Lei designed the study, analyzed the results and wrote the manuscript.

\section{CONFLICT OF INTEREST}

The authors don't have any conflicts of interest to declare.

\section{REFERENCES}

1. Dogruman-Al F, Kustimur S, Yoshikawa H, Tuncer C, Simsek Z, Tanyuksel M, Araz E, Boorom K. Blastocystis subtypes in irritable bowel syndrome and inflammatory bowel disease in Ankara, Turkey. Mem Inst Oswaldo Cruz 2016; 104: 724-727.

2. Lee H, Seo M, Oem J, Kim Y, Lee S, Kim J, Jeong H, Jheong W, Kim Y, Lee W, Kwon O, Kwak D. Molecular detection and subtyping of Blastocystis detected in wild boars (Sus scrofa) in South Korea. J Wildlife Dis 2020; 56: 662-666.

3. Yadav P, Khalil S, Mirdha BR. Molecular appraisal of intestinal parasitic infection in transplant recipients. Indian J Med Res 2016; 148: 258-263.

4. Rasti S, Hassanzadeh M, Hooshyar H, Momen-Heravi M, Mousavi SGA, Abdoli A. Intestinal parasitic infections in different groups of immunocompromised patients in Kashan and Qom cities, central Iran. Scand J Gastroenterol 2017; 52: 738-741.

5. Yakoob J, Jafri W, Beg MA, Abbas Z, Naz S, Islam M, Khan R. Ir- ritable bowel syndrome: Is it associated with genotypes of Blastocystis hominis. Parasitol Res 2010; 106: 1033-1038.

6. Maloney JG, Lombard JE, Urie NJ, Shivley CB, Santin M. Zoonotic and genetically diverse subtypes of Blastocystis in US preweaned dairy heifer calves. Parasitol Res 2019; 118: 575-582.

7. Alfellani MA, Jacob AS, Perea NO, Krecek RC, Taner-Mulla D, Verweij JJ, Levecke B, Tannich E, Clark CG, Stensvold CR. Diversity and distribution of Blastocystis sp. subtypes in non-human primates. Parasitology 2013; 140: 966-971.

8. Ramírez JD, Sánchez A, Hernández C, Flórez C, Bernal MC, Giraldo JC, Reyes P, López MC, García L, Cooper PJ. Geographic distribution of human Blastocystis subtypes in South America. Infection Genetics \& Evolution 2016; 41: 32-35.

9. Zanzani SA, Gazzonis AL, Epis S, Manfredi MT. Study of the gastrointestinal parasitic fauna of captive non-human primates (Macaca fascicularis). Parasitol Res 2016; 115: 307-312.

10. Zhao GH, Hu XF, Liu TL, Hu RS, Yu ZQ, Yang WB, Wu YL, Yu SK, Song JK. Molecular characterization of Blastocystis sp. In captive wild animals in Qinling Mountains. Parasitol Res 2017; 116: 2327-2333.

11. Stensvold CR, Clark CG. Current status of Blastocystis: A personal view. Parasitol Int 2016; 65: 763-771.

12. Alfellani MA, Stensvold CR, Vidal-Lapiedra A, Onuoha ESU, Fagbenro-Beyioku AF, Clark CG. Variable geographic distribution of Blastocystis subtypes and its potential implications. Acta Trop 2013; 126: 11-18.

13. Malheiros AF, Stensvold CR, Clark CG, Braga GB, Shaw JJ. Molecular characterization of Blastocystis obtained from members of the indigenous tapirape ethnic group from the Brazilian Amazon region, Brazil. Am J Trop Med Hyg 2011; 85: 1050-1053.

14. Deng L, Chai Y, Zhou Z, Liu H, Zhong Z, Hu Y, Fu H, Yue C, Peng G. Epidemiology of Blastocystis sp. infection in China: a systematic review. Parasite 2019; 26: 41.

15. Scicluna SM, Tawari B, Clark CG. DNA barcoding of Blastocystis. Protist 2006; 157: 77-85.

16. Lv XH, Chen XX, Yuan MQ, Cai WX, Qiao JY, Wu XM. The intestinal parasitic infection and its morphology of Rhinopithecus roxellanaes and Macaca mulatts. J Econ Anim 2010; 14: 92-95.

17. Vaisusuk K, Saijuntha W, Sedlak S, Thanchomnang T, Pilap W, Suksavate W, Stensvold CR, Tantrawatpan C. Blastocystis subtypes detected in long-tailed macaques in Thailand-further evidence of cryptic host specificity. Acta Trop 2018; 184: 78-82.

18. Helenbrook WD, Wade SE, Shields WM, Stehman SV, Whipps CM. Gastrointestinal parasites of Ecuadorian mantled howler monkeys (Alouatta palliata aequatorialis) based on fecal analysis. J Parasitol 2015; 101: 341-350.

19. Petrásová J, Uzlíková M, Kostka M, Petrželková KJ, Huffman MA, Modrý D. Diversity and host specificity of Blastocystis in syntopic primates on Rubondo Island, Tanzania. Int J Parasitol 2011; 41: 1113-1120.

20. Yoshikawa H, Wu Z, Pandey K, Pandey BD, Sherchand JB, Yanagi T, Kanbara H. Molecular characterization of Blastocystis isolates from children and rhesus monkeys in Kathmandu, Nepal. 
Vet Parasitol 2009; 160: 295-300.

21. Parkar U, Traub RJ, Vitali S, Elliot A, Levecke B, Robertson I, Geurden T, Steele J, Drake B, Thompson RCA. Molecular characterization of Blastocystis isolates from zoo animals and their animalkeepers. Vet parasitol 2010; 169: 8-17.

22. Zaman V, Ng GC, Suresh K, Yap EH, Singh M. Isolation of Blastocystis from the cockroach (Dictyoptera: Blattidae). Parasitol Res 1993; 79: 73-74.

23. Suresh K, Mak JW, Chuong LS, Ragunathan T, Init I. Sac-like pouches in Blastocystis from the house lizard Cosymbotus platyurus.
Parasitol Res 1997; 83: 523-525.

24. Farah Haziqah MT, Asyiqin MN, Mohd Khalid MN, Suresh K, Rajamanikam A, Chandrawathani P, Mohd Zain SN. Current status of Blastocystis in cockroaches. Trop Biomed 2017; 34: 741-745.

25. Deng L, Yao JX, Liu HF, Zhou ZY, Chai YJ, Wang WY, Zhong ZJ, Deng JL, Ren ZH, Fu HL, Yan X, Yue CJ, Peng GN. First report of Blastocystis in giant pandas, red pandas, and various bird species in Sichuan province, southwestern China. Int J Parasitol Parasites Wildl 2019; 9: 298-304. 
